Requisitos técnicos minimos: Procesador Pentium o superior. $32 \mathrm{Mb}$ RAM (recomendado $64 \mathrm{Mb}$ ). Windows $95,98, \mathrm{Me}$, 2000 , NT 4, XP, Linux. Unidad de CD-ROM.
DIGIBIB $\odot$ es un producto de

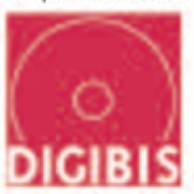

basado en ISBD/GARR, IBERMARC/MARC21 en un entorno XML diseñado específicamente para el intercambio de información en Internet.

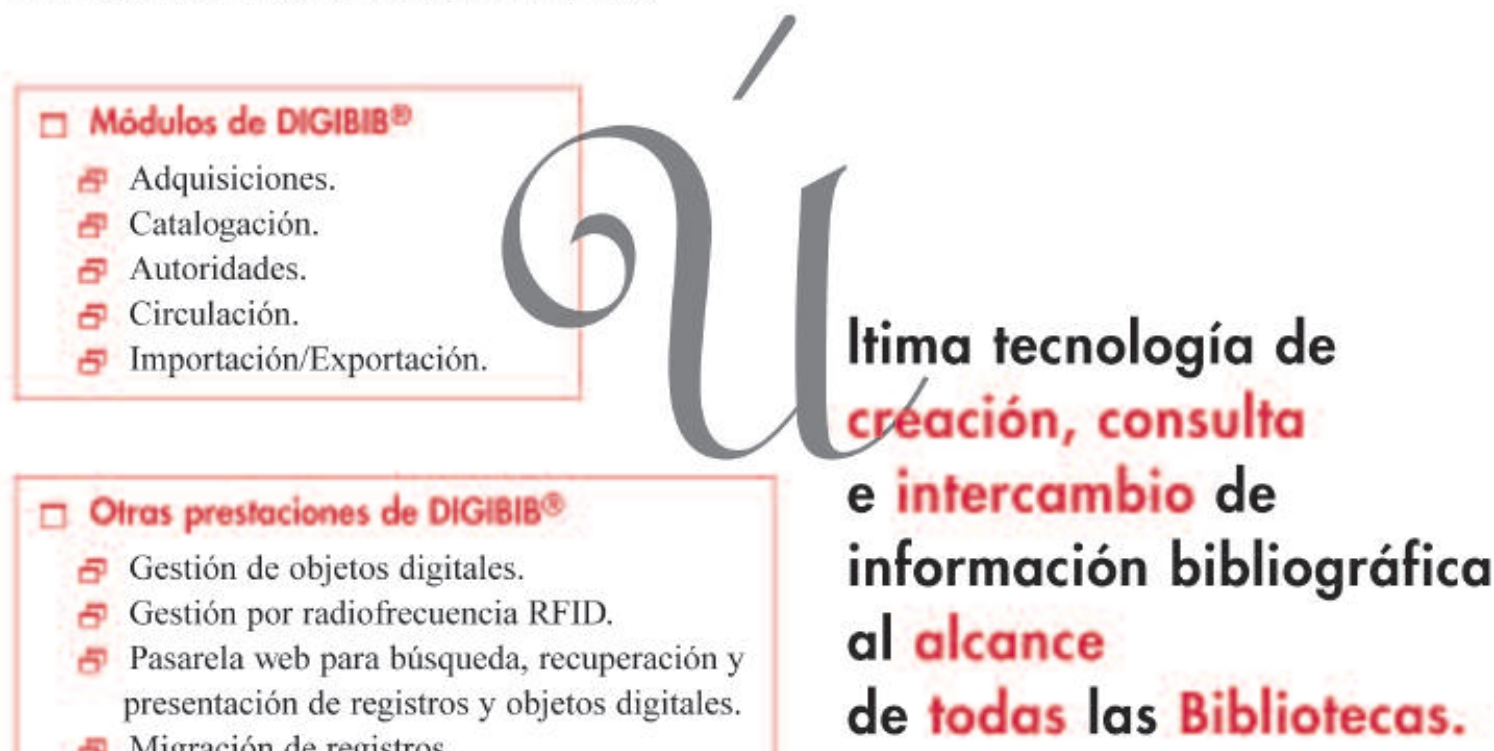

DoGIBIS, empresa especializada en el desarrollo de Bibliotecas Virtuales en colaboración con diversas instituciones, lidera el campo de la distribución de recursos electrónicos. En nuestro catálogo de publicaciones se pueden encontrar más de 2.000 obras digitalizadas.

\title{
Producción española con visibilidad internacional (ISI-WOS) en biblioteconomía y documentación (I)
}

\section{Por Grupo Scimago}

EL ANÁLISIS DE LA PRODUCCIÓN CIENTÍFICA de las diferentes áreas temáticas y disciplinas es un tema recurrente en los estudios bibliométricos. De todas las sometidas a análisis, una de las más estudiadas quizá sea la de biblioteconomía y documentación. Esto se debe no tanto a su importancia como disciplina en el contexto científico global, sino más bien a la proximidad al campo disciplinar de quienes realizan este tipo de análisis.

Sea por la razón que fuere, existen gran cantidad de estudios de la disciplina, tanto a nivel mundial como en lo que respecta a España. No es objetivo de esta breve nota realizar una completa revisión biblio- gráfica sobre la cuestión, sin embargo a modo de ejemplo destacaremos los estudios de White y McCain para la disciplina en el mundo ${ }^{1}$, y de Moya-Anegón y Herrero-Solana en el caso de la región iberoamericana ${ }^{2}$.

\section{«Dado el carácter} instrumental de la disciplina, existe producción LIS en revistas de otras categorías temáticas»

Uno de los aspectos determinantes en este tipo de estudio es la fuente de datos con la que se trabaja, la más utilizada es el ISI Web of Knowledge, de Thomson Scientific. Este recurso categoriza las revistas que recoge y cuenta con una sección temática denominada "Información science and library science" (conocida de forma abreviada como $L I S)$, que actualmente agrupa 54 títulos de revistas aunque en los últimos 15 años han pasado por la categoría más de 100 . En este trabajo analizaremos la producción española de la disciplina para el período 1995-2004. Nuestra fuente básica será el ya citado ISI Web of Knowledge. Los indicadores y representaciones presentadas proceden del Atlas de la ciencia.

\section{http://www.atlasofscience.net}

Todos los estudios de esta área que se basan en ISI utilizan la categoría LIS para seleccionar las revistas fuente. Sin embargo, algunos 


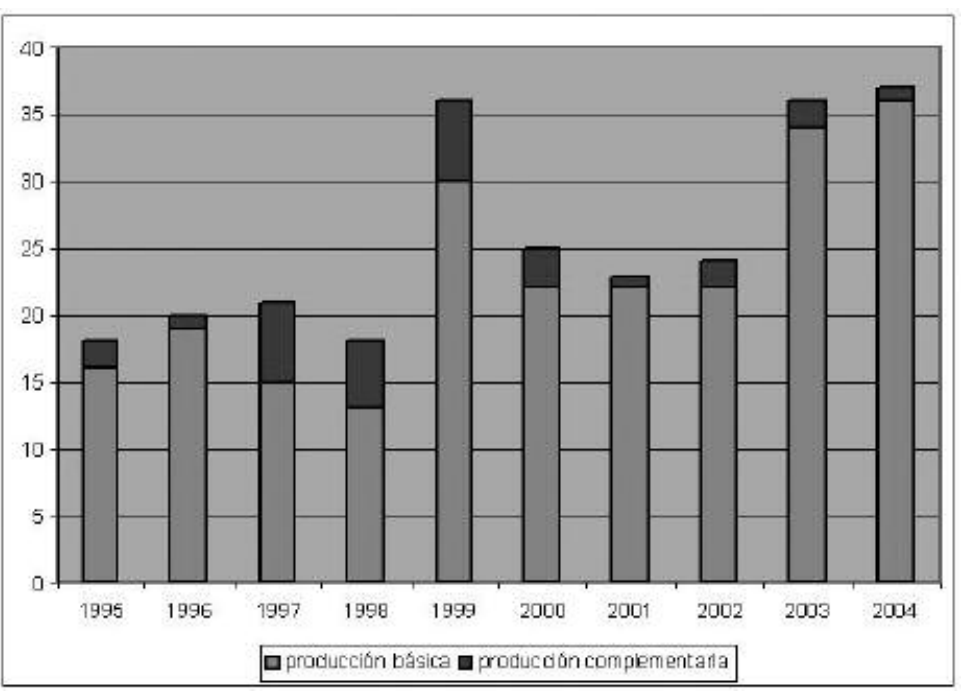

Figura 1

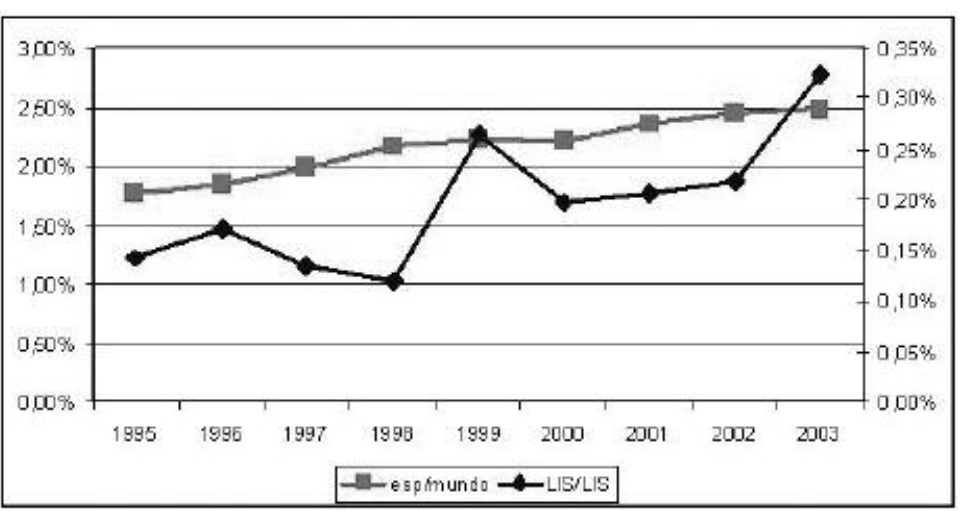

Figura 2

\begin{tabular}{|c|l|c|c|}
\hline & \multicolumn{1}{|c|}{ Institución } & Documentos & Citas \\
\hline 1 & Centro de Información y Documentación Científica & 39 & 138 \\
\hline 2 & Universidad de Granada & 50 & 99 \\
\hline 3 & Universidad de Alcalá de Henares & 16 & 67 \\
\hline 4 & Universidad Carlos III de Madrid & 29 & 49 \\
\hline 5 & Universidad de Extremadura & 19 & 49 \\
\hline 6 & Instituto Municipal Investigs. Médicas (Barcelona) & 4 & 35 \\
\hline 7 & Universidad Complutense de Madrid & 9 & 23 \\
\hline 8 & Universidad Politécnica de Cataluña & 7 & 15 \\
\hline 9 & Universidad Politécnica de Madrid & 10 & 14 \\
\hline 10 & Universidad de Jaén & 5 & 13 \\
\hline 11 & Universidad Autónoma de Madrid & 6 & 8 \\
\hline 12 & Instituto de Prospectiva Tecnológica & 4 & 6 \\
\hline 13 & Universidad Pública de Navarra & 4 & 5 \\
\hline 14 & Universidad de Oviedo & 4 & 3 \\
\hline 15 & Universidad Politécnica de Valencia & 8 & 2 \\
\hline 16 & Universidad de Salamanca & 5 & 2 \\
\hline 17 & Instituto de Salud Carlos III de Madrid & 5 & 2 \\
\hline 18 & Universidad de País Vasco & 0 \\
\hline 19 & Universidad de Navarra & 0 \\
\hline
\end{tabular}

Tabla 1 autores señalan que esto es insuficiente, ya que dado el carácter instrumental de la disciplina existe producción LIS en revistas de otras categorías temáticas. Jiménez Contreras plantea este problema, para cuya solución propone expandir este grupo de documentos mediante una serie de búsquedas en texto libre filtradas manualmente $^{3}$.

No obstante, este método no es demasiado preciso. Por ello, nos basamos en la idea de Glanzel, Schubert y Czerwon, que plantean la posibilidad de caracterizar la producción mediante sus citas ${ }^{4}$. De esta forma identificamos los trabajos de españoles que han publicado en revistas LIS, y a éstos añadimos los trabajos de españoles cuya categoría temática más citada ha sido LIS. Encontramos 229 registros para el primer grupo (producción básica) y 29 para el segundo (producción complementaria).

En la figura 1 se puede apreciar la evolución temporal de ambos grupos. Apreciamos una tendencia de crecimiento sostenido, aunque con ciertos altibajos motivados por lo escaso de la producción en el dominio. En la figura 2 podemos tener una idea más clara de cuál es el papel de la LIS con relación a España y el mundo. La línea clara, cuyo eje está a la derecha, nos indica el volumen que representa la producción científica total de España frente al mundo. En los últimos años ha crecido de forma sostenida, pasando del $1,77 \%$ al $2,45 \%$. La línea oscura representa la $L I S$, eje secundario, que si bien tiene una tasa de crecimiento mayor llega a representar solo el $0,32 \%$ de la producción LIS del mundo. Esto nos indica un potencial terreno de crecimiento para la disciplina en España para los próximos años.

Por último, finalizaremos esta primera parte indicando de qué manera se distribuye la producción en función de las instituciones. En la tabla 1 encontramos aquellas que tienen al menos 4 documentos publicados en el período. En la tabla se presentan dos indicadores de interés: el primero es la producción total, mientras que el segundo son las citas recibidas por esa misma producción. En cuanto a la ordenación de la tabla, hemos decidido hacerla por el segundo valor, ya que brinda una idea más 
completa de la visibilidad científica de cada institución.

De esta forma tenemos en primer lugar al Cindoc que, a pesar de ser la segunda institución en términos de producción, es la que recoge más citas y tiene más visibilidad. La segunda de la lista es la Universidad de Granada, que tiene la mayor producción, pero cuyos trabajos no recogen la misma citación que la anterior. La tercera es la Universidad de Alcalá de Henares, cuya producción es pequeña pero muy citada. Luego siguen la Carlos III y Extremadura, con la misma cantidad de citas, aunque la segunda con una producción menor. Por último, es importante destacar el Instituto Municipal de Investigaciones Médicas de Barcelona, que pese a te- ner una producción muy modesta, en el área recibe una gran citación. La mayor parte de la producción se ha canalizado a través de revistas médicas como Medicina clínica y en colaboración con el Cindoc.

Sobre las fuentes en las que se ha publicado, así como la literatura citada y los autores más productivos, hablaremos en la segunda entrega de esta nota.

\section{Notas}

1. White, Howard D.; Katherine W. Mccain "Visualizing a discipline: an author co-citation analysis of information science, 1972-1995".

En: Journal of the American Society for Information Science, 1998 , v. 49, n. 4, pp. 327-355.

2. Moya-Anegón, Félix; Herrero-Solana, Víctor. "Visibilidad internacional de la producción científica iberoamericana en bibliotecología y documentación (1991-2000)". En: Ciência da informação, 2002, v. 31, n. 3, pp. 54-65.
3. Jiménez-Contreras, Evaristo. "La aportación española a la producción científica internacional en biblioteconomía y documentación: balance de diez años (1992-2001)". En: BiD: biblioteconomía y documentació, 2002, desembre, n. 9.

http://www.ub.es/biblio/bid/09jimen2.htm

4. Glanzel, W.; Schubert, A.; Czerwon, H. J.

"An item-by-item subject classification of papers Publisher in multidisciplinary and general journal using reference analysis". En: Scientometrics, 1999 , v. 44, n. 3, pp. 427-439.

5. Moya-Anegón, Félix (dir.); Chinchilla-Rodríguez, Zaida (coord.). Indicadores bibliométricos de la actividad científica española-2004. Madrid: Fundación Española de Ciencia y Tecnología, 2005. (Observatorio Fecyt de Política Científica y Tecnología).

Informe enviado a EPI por el Grupo SCImago (Imago scientiae o visualización de la ciencia).

scimago@ugr.es

http://scimago.ugr.es

http://www.atlasofscience.net 